\title{
Vector Image Model to Object Boundary Detection in Noisy Images
}

\author{
S.Suryakala ${ }^{1}$, I.V.Sushmitha Dani ${ }^{2}$, I.Shibiya Sherlin ${ }^{3}$, S.Sheba Monic ${ }^{4}$, A.Sushma Thavakumari ${ }^{5}$, Christo Ananth ${ }^{6}$ \\ U.G. Scholars, Department of ECE, Francis Xavier Engineering College, Tirunelveli ${ }^{1,2,3,4,5}$ \\ Associate Professor, Department of ECE, Francis Xavier Engineering College, Tirunelveli ${ }^{6}$
}

\begin{abstract}
A New model is designed for boundary detection and applied it to object segmentation problem in medical images. Our edge following technique incorporates a vector image model and the edge map information. The proposed technique was applied to detect the object boundaries in several types of noisy images where the ill-defined edges were encountered. The proposed techniques performances on object segmentation and computation time were evaluated by comparing with the popular methods, i.e., the ACM, GVF snake models. Several synthetic noisy images were created and tested. The method is successfully tested in different types of medical images including aortas in cardiovascular MR images, and heart in CT images.
\end{abstract}

\section{Index Terms- Noisy Images, ACM, GVF snake models}

\section{INTRODUCTION}

Image analysis is the extraction of meaningful information from images; mainly from digital images by means of digital image processing techniques. Image analysis tasks can be as simple as reading bar-coded tags or as sophisticated as identifying a person from their face. Image analysis operations produce numerical or graphical information based on characteristics of the original image. They break into objects and then classify them. They depend on the image statistics. Common operations are extraction and description of scene and image features, automated measurements, and object classification. Image analyse are mainly used in machine vision applications. Computer image analysis largely contains the fields of computer or machine vision, and medical imaging, and makes heavy use of pattern recognition, digital geometry, and signal processing. It is the quantitative or qualitative characterization of two-dimensional (2D) or three-dimensional (3D) digital images. 2D images are, for example, to be analyzed in computer vision, and 3D images in medical imaging.

Image compression and decompression reduce the data content necessary to describe the image. Most of the images contain lot of redundant information, compression removes all the redundancies. Because of the compression the size is reduced, so efficiently stored or transported. The compressed image is decompressed when displayed. Lossless compression preserves the exact data in the original image, but Lossy compression does not represent the original image but provide excellent compression. Image compression may be lossy or lossless. Lossless compression is preferred or archival purposes and often for medical imaging, technical drawings, clip art, or comics. Lossy methods are especially suitable for natural images such as photographs in applications where minor loss of fidelity is acceptable to achieve a substantial reduction in bit rate.

Methods for lossless image compression are:

* Run-length encoding - used as default method in PCX and as one of possible in BMP, TGA, TIFF

* DPCM and Predictive Coding

* Entropy encoding

* Adaptive dictionary algorithms such as LZW - used in GIF and TIFF

* Deflation - used in PNG, MNG, and TIFF

* Chain codes

Methods for lossy image compression:

* Reducing the color space to the most common colors in the image. The selected colors are specified in the color palette in the header of the compressed image. Each pixel just references the index of a color in the color palette. This method can be combined with dithering to avoid posterization.

* Chroma sub sampling. This takes advantage of the fact that the human eye perceives spatial changes of brightness more sharply than those of color, by averaging or dropping some of the chrominance information in the image.

* Transform coding. This is the most commonly used method. A Fourier-related transform such as DCT or the wavelet transform are applied, followed by quantization and entropy coding.

* Fractal compression.

In [1], Bing Li and Scott T. Acton proposed a novel static external force for active contours, called the vector field convolution. The VFC field is calculated by convolving a vector field kernel with the edge map generated from the image. Different external forces have been proposed to improve the performance of snakes. The external forces can be generally classified as dynamic forces and static forces. The dynamic forces are those that depend on the snake and, as 
International Journal of Advanced Research in Management, Architecture, Technology and Engineering (IJARMATE)

a result, change as the snake deforms. The static forces are those that are calculated from the image, and remain unchanged as the snake de-forms. The main disadvantage of this method id only high capture range images can be used here.

In [2], Chenyang Xu and Jerry L. Prince proposed a method called gradient vector flow (GVF). The gradient vector flow fields are dense vector fields derived from images by minimizing certain energy functional in a variation framework. The minimization is achieved by solving a pair of decoupled linear partial differential equations that diffuse the gradient vectors of a gray-level or binary edge map computed from the image. The GVF snake also has a large capture range, which means that, barring interference from other objects, it can be initialized far away from the boundary. The disadvantage of the model is resultant value is only closer not accurate.

In [3], Chenyang $X u$ and Jerry L. Prince presents a new class of external forces for active contour model gradient vector flow (GVF) fields, are dense vector fields derived from images by minimizing energy functional in a variation framework. The minimization is achieved by solving a pair of decoupled linear partial differential equations which diffuses the gradient vectors of a gray-level or binary edge map computed from the image. Particular advantages of the GVF snake over a traditional snake are its insensitivity to initialization and ability to move into concave boundary regions. A new external force model for snakes called gradient vector flow (GVF). The disadvantage of the method is performance degradation.

In [4], François Destrempes, Gilles Soulez and Guy Cloutier proposed a new semi-automatic segmentation method. The intima-media thickness (IMT) is a double-line pattern visualized by echography on both walls of the common carotid arteries in a longitudinal image. It is formed by two parallel lines, which consist of the leading edges of two anatomical boundaries: the lumen-intima and media-adventitia interfaces. Semi-automatic segmentation of the IMT on the far wall of carotid arteries in $B$-mode ultrasound images is a useful tool for clinical applications. Rayleigh distributions have been used to model the local brightness of the speckle pattern in a $B$-mode image. The main disadvantage of this method is only high contrast image can be used here.

In [5], FrCdCric Leymarie and Martin D. Levine proposed a explored a new segmentation technique Snakes can be represented as energy-minimizing splines guided by external constraint forces and image forces such as lines, edges, subjective contours, and region homogeneities found in the image. Furthermore, internal spline forces impose smoothness constraints on the modeled contours. By combining and integrating various types of information found in an image, snakes can lead to results that are at least comparable with other image-segmentation techniques. The steady-state criterion for terminating the optimization procedure is an overly strong one, leading to unnecessary oscillations and, possibly, the snake receding from a valid solution. The drawback of the model is, the model contains multiple processes.

\section{PROPOSED SYSTEM}

Our proposed system is edge following technique for boundary detection in noisy images. Utilization of the proposed technique is exhibited via its application to various types of medical images. Our proposed technique can detect the boundaries of objects in noisy images using the information from the intensity gradient via the vector image model and the texture gradient via the edge map. The performance and robustness of the technique have been tested to segment objects in synthetic noisy images and medical images including prostates in ultrasound images, left ventricles in cardiac magnetic resonance images, aortas in cardiovascular MR images, and knee joints in computerized tomography images.

It gives more information for searching the boundary of objects and increases the probability of searching the correct boundary. The magnitude and direction of the average edge vector field give information of the boundary which flows around an object. In addition, the edge map gives information of edge which may be a part of object boundary. Hence, both average edge vector field and edge map are exploited in the decision of the edge following technique.

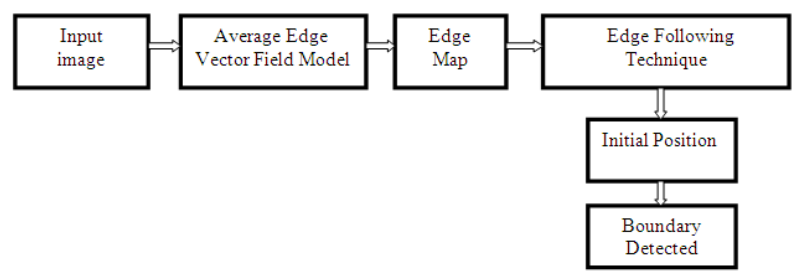

Fig.1. Overall System Design

The overall system design of the Edge following technique using the average edge vector field and edge map method is shown in the figure 5.1 with the deterministic annealing and nonexpansive mapping. It gives more information for searching the boundary of objects and increases the probability of searching the correct boundary.

The results of detecting the object boundaries in noisy images show that the proposed technique is much better than the five contour models. The results of the running time on several sizes of images also show that our method is more efficient than the five contour models. 


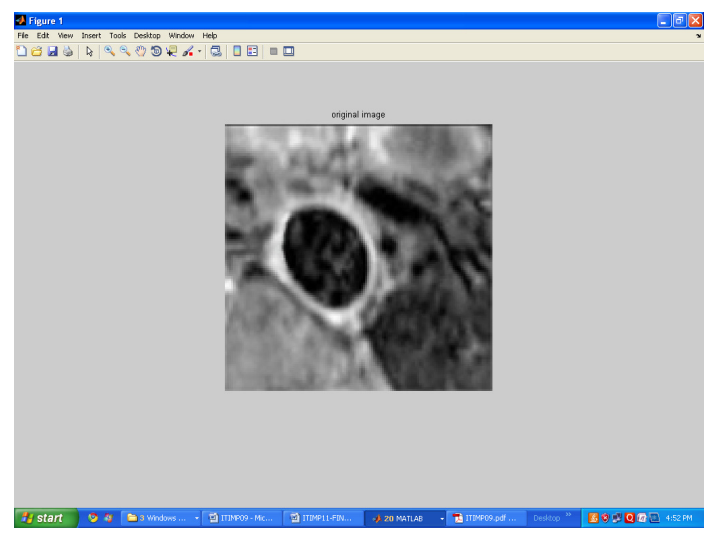

Fig.2. Input image

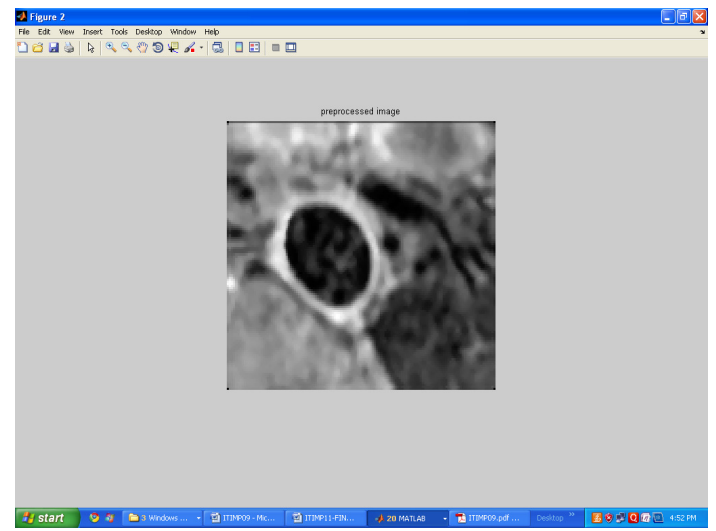

Fig.3. Preprocessed image

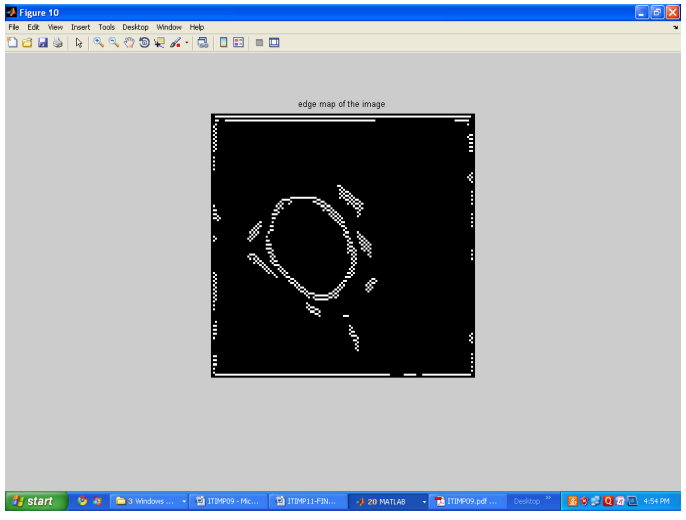

Fig.4. Edge map of the image

\section{CONCLUSION}

A New model is designed for boundary detection and applied it to object segmentation problem in medical images. Our edge following technique incorporates a vector image model and the edge map information. The proposed technique was applied to detect the object boundaries in several types of noisy images where the ill-defined edges were encountered. The proposed techniques performances on object segmentation and computation time were evaluated by comparing with the popular methods, i.e., the ACM, GVF snake models. Several synthetic noisy images were created and tested. The method is successfully tested in different types of medical images including aortas in cardiovascular MR images, and heart in CT images.

\section{REFERENCES}

1. Bing Li and Scott Acton T. (2013), 'Active contour external force using vector field convolution for image segmentation', IEEE Transactions on Image Processing, Vol. 16, No. 8, pp. 2096-2106.

2. Chenyang Xu and Jerry Prince L. (2011), 'Snakes, shapes, and gradient vector flow', IEEE Transactions on Image Processing, Vol. 7, No. 3, pp. 359-369.

3. Chenyang Xu and Jerry Prince L. (2010), 'Gradient vector flow: A new external force for snake', in IEEE Proceedings on Conference Computation Visual Pattern Recognition, pp. 66-71.

4. François Destrempes, Gilles Soulez and Guy Cloutier (2009), 'Segmentation in ultrasonic B-mode images of healthy carotid arteries using mixtures of Nakagami distributions and stochastic optimization', IEEE Transactions on Image Processing, Vol. 28, No. 2, pp. 215-229.

5. FrCdCric Leymarie and Martin Levine D. (2008), 'Tracking deformable objects in the plane using an active contour model', IEEE Transactions on Pattern Analysis and Machine Intelligence, Vol. 15, No. 6, pp. 617-634. 